Article

\title{
Implementation of MALDI-TOF Mass Spectrometry and Peak Analysis: application to the discrimination of Cryptococcus species and their interspecies hybrids
}

\author{
Margarita E. Zvezdanova 1,2, Manuel J. Arroyo ${ }^{3}$, Gema Méndez ${ }^{3}$, Jesús Guinea 1, 2, 4, Luis Mancera \\ 3, Patricia Muñoz 1,2, 4, 5, Belén Rodríguez-Sánchez ${ }^{1,2 *}$, and Pilar Escribano 1,2, \\ 1 Clinical Microbiology and Infectious Diseases Department, Hospital General Universitario Gregorio \\ Marañón, Madrid, Spain. Doctor Esquerdo, 46. 28007 Madrid, Spain. estrellanik zv@hotmail.es (M.E.Z), \\ jguineaortega@yahoo.es (J.G.), pmunoz@hggm.es (P. M.), mbelen.rodriguez@iisgm.com (B.R.S.), \\ pilar.escribano.martos@gmail.com (P.E.) \\ 2 Instituto de Investigación Sanitaria Gregorio Marañón, Madrid, Spain. \\ 3 Clover Bioanalytical Software, Av. de la Innovación, 118016 Granada, Spain \\ manuel.arroyo@cloverbiosoft.com (M.J.A.), gema.mendez@cloverbiosoft.com (G.M.), \\ luis.mancera@cloverbiosoft.com (L.M.) \\ 4 CIBER de Enfermedades Respiratorias (CIBERES CB06/06/0058), Madrid, Spain. \\ 5 Medicine Department, School of Medicine, Universidad Complutense de Madrid, Madrid, Spain \\ * Correspondence: mbelen.rodriguez@iisgm.com; Tel.: (+34 914269595; Doctor Esquerdo, 46. 28007 Madrid, \\ Spain
}

\begin{abstract}
MALDI-TOF (Matrix-Assisted Laser Desorption/Ionization Time-of Flight) is a type of mass spectrometry (MS) that has been widely implemented for the rapid identification of microorganisms over the last decade. The accuracy and flexibility of this method has encouraged researchers to implement the analysis of protein spectra obtained by MALDI-TOF for the discrimination of close-related species and bacterial typing. In this study, a standardized methodology based on the detection of species-specific protein peaks from the spectra obtained with MALDI-TOF is described. The methodology was applied to a collection of Cryptococcus spp. $(\mathrm{n}=70)$ previously characterized by Amplified Fragment Length Polymorphism (AFLP) and sequencing of the ITS1-5.8S-ITS2 region. An expanded ad-hoc database was also built for their discrimination with MALDI-TOF. This approach did not allow the discrimination of the interspecies hybrids. However, the performance of peak analysis with the application of the PLS-DA and SVM algorithms in a two-step analysis allowed $96.95 \%$ and $96.55 \%$ correct discrimination of C. neoformans from the interspecies hybrids, respectively. Besides, PCA analysis prior to SVM provided $98.45 \%$ correct discrimination of the 3 analyzed species in a one-step analysis. The method is cost-efficient, rapid and user-friendly. The procedure can also be automatized for an optimized implementation in the laboratory routine.
\end{abstract}

Keywords: Cryptococcus spp; MALDI-TOF MS, peak analysis, in-house library, hierarchical clustering 


\section{Introduction}

The genus Cryptococcus has classically comprised two sibling species with great importance from the clinical point of view: Cryptococcus neoformans and C. gattii, the causative agents of cryptococcosis. Whilst $C$. neoformans complex has been associated with meningitis in immunosuppressed patients, $C$. gatti has been shown to cause disease in both immune competent and immunocompromised population [1,2]. Species differentiation is important in order to establish the epidemiology, virulence and susceptibility pattern to the commonly used antifungal drugs [3-6]. Traditionally, species assignment has been achieved by morphology analysis of the colonies grown on specific culture media and serological tests [7]. The availability of DNA-based methodologies as restriction fragment length polymorphism (RFLP) analysis [8], amplified fragment length polymorphism (AFLP) analysis [9], multilocus microsatellite typing -MLMT-[10], and multilocus sequence typing -MLST- [11] has allowed the identification of Cryptococcus species and molecular types in the last years $[12,13]$. Genotyping methods have identified the following major molecular types: AFLP1/VNI, AFLP1A, AFLP1B/VNII for C. neoformans; AFLP2/VNIV for C. deneoformans, AFLP3/VNIII for the interspecies hybrid C. neoformans neoformans $\times$ C. deneoformans; and AFLP4/VGI, AFLP5/VGIII, AFLP6/VGII, AFLP7/VGIV and AFLP10/VGIV, VGII for C. gattii complex [14, 15]. Molecular techniques have shown to be accurate and robust although the whole procedure is cumbersome, time consuming, and delays the final identification. Although genomic analysis is currently the gold standard for Cryptococcus spp. identification, its high requirements in hands-on time and expertise has led to the evaluation of alternative tools.

Matrix-assisted laser desorption ionization-time of flight mass spectrometry (MALDI-TOF MS) has emerged as a promising technology for the rapid and reliable identification of yeasts [16-18]. Isolates belonging to the Candida genus have been shown to be easily identified at the species level either from single colonies or directly from clinical samples using MALDI-TOF MS [19]. However, non-Candida yeasts still represent a challenge for this technology, especially when trying to identify genera poorly represented or even lacking in the commercial databases [20]. In this case, expanded in-house databases containing protein spectra from the underrepresented species and genera have shown to overcome this drawback [16]. Although this approach has worked before for the discrimination between $C$. neoformans and $C$. gatti complexes [21, 22], the available information about MALDI-TOF discrimination within the C. neoformans complex is still limited [23].

In this study, MALDI-TOF has been applied for the discrimination between $C$. deneoformans, $C$. neoformans and the interspecies hybrids. For this purpose, a "classical" approach was applied: a database was built using well-characterized isolates for the identification of the Cryptococcus spp. isolates using the Biotyper system developed by Bruker Daltonics (Bremen, Germany). Besides, the protein spectra from these isolates were processed and classified using different algorithms in order to find species-specific peaks that allowed their differentiation.

\section{Materials and Methods}

\section{Isolates}

We retrospectively selected 70 Cryptococcus spp. isolates from clinical samples $(\mathrm{n}=70)$ belonging to 67 patients admitted to Hospital Gregorio Marañón (Madrid, Spain) from 1994 to 2007. Isolates sourced from cerebrum spinal fluids (51\%), blood (33\%), respiratory samples $(10 \%)$, and others $(6 \%)$. They were morphologically identified on Columbia agar $+5 \%$ sheep blood plates (Biomérieux Marcy L'étoile, France) at $35^{\circ} \mathrm{C}$, and by means of the ID $32 \mathrm{C}$ system (bioMérieux, Marcy l'Etoile, France). All isolates were stored at $-80^{\circ} \mathrm{C}$ in water until further analysis.

\section{Molecular identification}

To ensure the purity, the isolates were grown on Columbia agar supplemented with $5 \%$ of sheep blood plates and incubated at $35^{\circ} \mathrm{C}$ for $24 \mathrm{~h}$. All isolates were previously identified by DNA 
sequencing analysis of the ITS1-5.8S-ITS2 region [24] and AFLP analysis [25]. Molecular identifications were considered as the reference in our study.

\section{Database construction}

Twenty-six Cryptococcus isolates - C. neoformans $(n=12)$, interspecies hybrids $(n=10)$ and $C$. deneoformans $(\mathrm{n}=4)$ - were processed according to the manufacturer's instructions and added to the in-house database (HGM library) as individual Main Spectra (MSPs).

The procedure for adding new entries to an in-house library has already been described [26]. Briefly, the instrument was calibrated before spectra acquisition using freshly prepared BTS; Cryptococcus isolates were processed as explained below and then spotted onto eight positions in the MALDI target plate and each position was read three times. Twenty-four protein spectra were thus achieved, 20 of which had to be identical in order to be accepted by the software (Biotyper, Bruker Daltonics, Bremen, Germany) as a MSP and added to the extended library.

\section{MALDI-TOF identification}

Forty-four Cryptococcus spp. isolates were blindly analysed using an LT Microflex benchtop MALDI-TOF mass spectrometer (Bruker Daltonics) for spectra acquisition, using default settings. For the identification of the protein spectra, the updated BDAL database containing $8223 \mathrm{MSPs}$ (Bruker Daltonics) was applied. This database contains 12 reference MSPs from C. neoformans and 7 from $C$. deneoformans. Besides, the expanded in-house HGM library developed in this study was used in combination with the commercial database.

The sample processing method applied consisted of a mechanical disruption step followed by a standard protein extraction. Briefly, a few colonies were picked, re-suspended in $300 \mu \mathrm{l}$ water HPLC-grade (High-Pressure Liquid Chromatography) and $900 \mu \mathrm{l}$ ethanol, and submitted to $5 \mathrm{~min}$ vortexing. After a brief spin, the supernatant was discarded and the pellet allowed drying completely at RT. Standard protein extraction with formic acid and acetonitrile was performed and $1 \mu \mathrm{l}$ of the supernatant was spotted onto the MALDI target plate in duplicates. Once the spots were dry, they were covered with $1 \mu$ l HCCA matrix (Bruker Daltonics), prepared following the manufacturer's instructions (Figure 1).

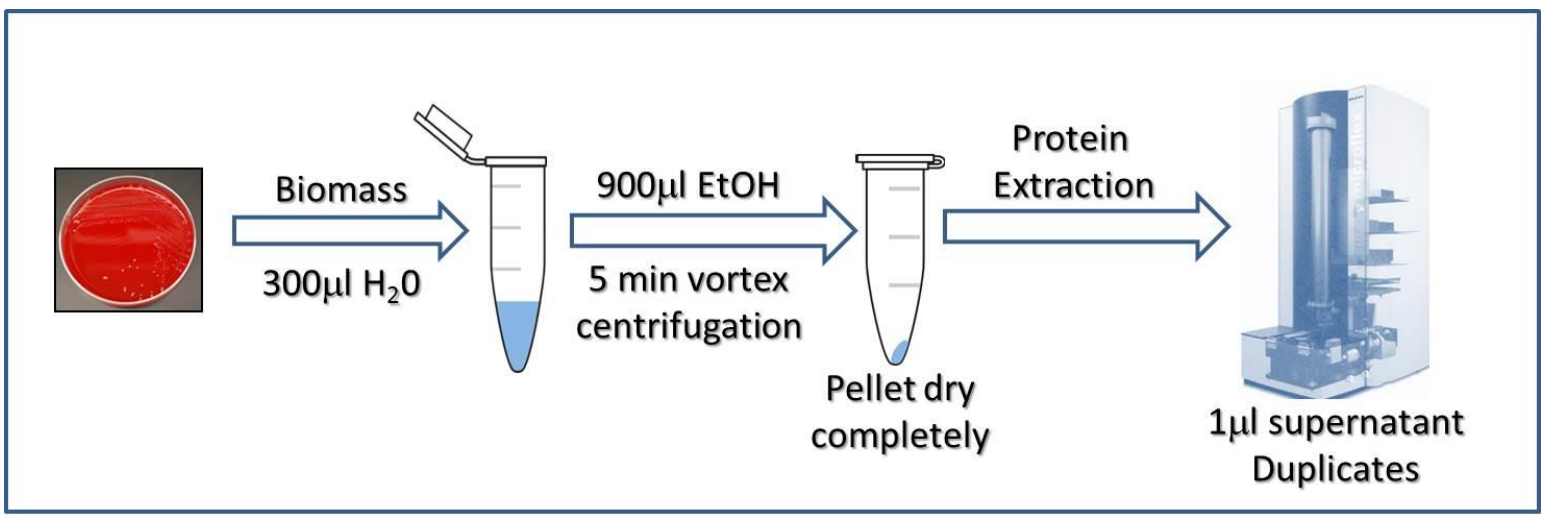

Figure 1. Workflow of the sample preparation method used in this study to obtain proteins from Cryptococcus spp. isolates for their identification by MALDI-TOF MS.

The identifications provided by MALDI-TOF MS were compared at the species level with those provided by AFLP analysis regardless of their score value (Table 1). Besides, score values $\geq 2.0$ were considered as "high-confidence" scores and those $\geq 1.7$ as "low-confidence" ones. Score values below 1.6 were only considered when consistent over the four top identifications, otherwise they were considered as "not reliable". 


\section{Peak Analysis}

For the classification of the three Cryptococcus species, their protein spectra were processed using Clover MS Data Analysis software (Clover Biosoft, Granada, Spain) with the parameters shown in Table S1 in order to achieve a peak matrix with a representative mass list in the range $2400 \mathrm{~m} / \mathrm{z}$ to $12000 \mathrm{~m} / \mathrm{z}$. Furthermore, spectra alignment was performed. First, the replicates from the same isolate were aligned in order to get an average spectrum. Finally, all average spectra were aligned together.

The rate of presence for the biomarker peaks was calculated for each species and then compared among species. Receiver Operating Characteristic (ROC) curve with Area under the Curve -AUChigher than 0.99 were used as quality indicators to measure the sensibility and specificity of a selected biomarker.

Once the putative biomarkers were selected and analyzed, a peak matrix was built containing all the aligned spectra from all Cryptococcus isolates, processed as described in Table S2. This peak matrix was constructed with ten species-specific biomarkers and it was used as input for a dendrogram obtained measuring Euclidean distance from Principal Component Analysis (PCA) scores.

Over the peak matrix, two approaches were applied in order to discriminate the three Cryptococcus species. The first one was a two-step method in which the discrimination of $C$. deneoformans from the other two species was performed as a first step and it was replicated by means of two supervised machine learning algorithms on the same peak matrix: Partial Least Squares Discriminant Analysis (PLS-DA) and Support Vector Machine (SVM). Results were validated using k-fold cross validation method.

In the second step, a new peak matrix was performed in order to achieve a better discrimination of C. neoformans from the interspecies hybrids. A second dendrogram was performed using the above mentioned parameters. Again, PLS-DA and SVM were applied to this second peak matrix to replicate the classification. The k-fold cross validation method was also applied. The two-step method was further improved by the exclusion from the peak matrix of peaks that did not provide enough discrimination.

Finally, in order to simplify the workflow, a one-step method was assayed so that the capacity of the algorithms to discriminate the three Cryptococcus species at the same time was tested. In this case, only one peak matrix with spectra from the three species was built and 5 species-specific biomarkers were included. The alignment and processing parameters were the same as in the two-steps approach. The one-step method was evaluated using the peak matrix generated as input data for PLS-DA and SVM analysis. Besides, the validation in both cases was performed using $\mathrm{k}$-fold confusion matrix.

\section{Ethic Statement}

The hospital Ethics Committee approved this study and gave consent for its performance (Code: MICRO.HGUGM.2017-003). Since only microbiological samples were analyzed, not human products, all the conditions to waive the informed consent have been met.

\section{Results}

\subsection{Molecular identification}

Genotyping of the isolates detected three different genotypes. The most common genotype was AFLP1/1B (C. neoformans, $n=34 ; 49 \%$ ), followed by AFLP3 (interspecies hybrids, $n=29 ; 41 \%$ ) and AFLP2 (C. deneoformans, $\mathrm{n}=7 ; 10 \%)$.

\subsection{Identification using MALDI-TOF MS}


The application of MALDI-TOF MS and the commercial database allowed the correct identification of $18 / 22$ C. neoformans isolates $(81.8 \%)$ and $1 / 3$ C. deneoformans isolates $(33.3 \%)$; the remaining $C$. neoformans isolates $-\mathrm{n}=4$ - could not be reliably identified and for $2 C$. deneoformans isolates MALDI-TOF did not provide species identification (Table 1). The identification of the interspecies hybrids $(n=19)$ was not achieved using the commercial database due to the lack of representation of this microorganism. These isolates were identified as C. neoformans complex in 9 cases (score $\geq 2.0, \mathrm{n}=7$; score $>1.7, \mathrm{n}=1$; score $<1.6, \mathrm{n}=1$ ), as $C$. deneoformans in 7 cases (score $>1.7, \mathrm{n}=4$; score $<1.6, \mathrm{n}=3$ ) and as $\mathrm{C}$. neoformans in 3 cases (score $>1.7$ ) -Table 1 -.

Only two isolates $(8.0 \%)$ were correctly identified at the species level with high-confidence score values $(\geq 2.0)$ whilst $52.3 \%$ of the samples -23 - were identified with low-confidence scores $(>1.7)$ -Table 1-. Another 4 isolates were reliably identified to the species level, although with scores values ranging between 1.7 and 1.6 and, finally, 8 isolates obtained scores below 1.6. The latter can be considered as unreliable identifications.

Table 1. Identification of Cryptococcus neoformans, C. deneoformans and interspecies hybrids using MALDI-TOF MS and the Biotyper library alone or in combination with the in-house HGM database.

\begin{tabular}{|c|c|c|c|c|c|c|c|c|}
\hline \multirow[t]{2}{*}{$\begin{array}{l}\text { IDENTIFICATION BY } \\
\text { DNA SEQUENCING }\end{array}$} & \multirow[t]{2}{*}{$\begin{array}{l}\text { Isolates } \\
\text { analyzed }\end{array}$} & \multicolumn{4}{|c|}{$\begin{array}{c}\text { IDENTIFICATION USING } \\
\text { THE BIOTYPER } \\
\text { DATABASE WITH } 8223 \\
\text { MSPS }\end{array}$} & \multicolumn{3}{|c|}{$\begin{array}{l}\text { IDENTIFICATION } \\
\text { USING THE } \\
\text { BIOTYPER } \\
\text { DATABASE WITH } \\
\text { 8223 MSPS + HGM } \\
\text { LIBRARY }\end{array}$} \\
\hline & & $\begin{array}{l}\text { Score } \\
\geq 2.0\end{array}$ & $\begin{array}{l}\text { Score } \\
\geq 1.7\end{array}$ & $\begin{array}{l}\text { Score } \\
\geq 1.6\end{array}$ & $\begin{array}{c}\text { Score } \\
<1.6\end{array}$ & $\begin{array}{l}\text { Score } \\
\geq 2.0\end{array}$ & $\begin{array}{l}\text { Score } \\
\geq 1.7\end{array}$ & $\begin{array}{l}\text { Score } \\
\geq 1.6\end{array}$ \\
\hline Cryptococcus neoformans & 22 & 2 & 13 & 3 & 4 & 18 & 4 & 0 \\
\hline Cryptococcus deneoformans & 3 & 0 & $2^{1}$ & 1 & 0 & 3 & 0 & 0 \\
\hline Interspecies hybrids & 19 & $7^{2}$ & $8^{3}$ & 0 & $4^{4}$ & 7 & $12^{5}$ & 0 \\
\hline TOTAL & 44 & 9 & 23 & 4 & 8 & 28 & 16 & 0 \\
\hline
\end{tabular}

${ }^{1}$ Identified as C. neoformans complex $(\mathrm{n}=2) ;{ }^{2}$ Identified as C. neoformans complex $(\mathrm{n}=7) ;{ }^{3}$ Identified as C. neoformans complex $(\mathrm{n}=1), \mathrm{C}$. deneoformans $(\mathrm{n}=4)$ and C. neoformans $(\mathrm{n}=3)$; ${ }^{4}$ Identified as $\mathrm{C}$. neoformans complex $(\mathrm{n}=1)$ and $C$. deneoformans $(\mathrm{n}=3)$; ${ }^{5}$ Identified as $C$. neoformans $(\mathrm{n}=7)$

Using the in-house library all $C$. neoformans and $C$. deneoformans isolates were correctly identified by MALDI-TOF MS at the species level (Table 1). Moreover, 21/25 of these isolates (84.0\%) were identified with score values $\geq 2.0$ which indicates a high-confidence level. The reliability of the identification was further demonstrated by the fact that the top $4-5$ identifications were identical in all cases. In all but two cases these top reference isolates belonged to the HGM in-house library.

However, the implementation of the expanded HGM library only allowed the correct identification of 12/19 interspecies hybrids, 7 of them with score values above 2.0. The high closeness of the interspecies hybrids with the other two Cryptococcus species made it difficult for MALDI-TOF MS to discriminate among them and misidentified 7 interspecies hybrids as $C$. neoformans (Table 1 ).

\subsection{Peak Analysis}


To improve the identification of the interspecies hybrids and their discrimination from $C$. deneoformans and $C$. neoformans, peak analysis was performed. The search for species-specific biomarker peaks yielded a list of 10 peaks that allowed the differentiation of the Cryptococcus species analysed, with 5 of them showing higher discriminative power (Table 2). The two-step method allowed correct differentiation of the interspecies hybrids which clustered distinctly in the dendrograms built using two different hierarchical clustering variations (Figure 2 and Figure S2). These dendrograms showed three different clusters where $C$. deneoformans isolates were clearly separated from C. neoformans and the interspecies hybrids. Accurate differentiation among the 3 Cryptococcus species was achieved using the peak matrix built upon the 5 most discriminative peaks, with only one spectrum from an interspecies hybrid misallocated in the C. neoformans cluster (Figure 2B). C. neoformans and the interspecies hybrids showed close relatedness between them based on their protein spectra.

The validation of the method yielded a $k$-fold $(k=10)$ score of $96.92 \%$ for PLS-DA performed over the peak matrix with 10 biomarkers and $98.46 \%$ for the analysis with 5 biomarkers. However, SVM algorithm achieved 100\% discrimination in both cases when PCA was applied (Table S3).

A second dendrogram was performed using hierarchical clustering analysis. It showed two well-defined clusters for C. neoformans and the interspecies hybrids (Figure S2). In this step only the 3 biomarkers to differentiate $C$. deneoformans from interspecies hybrids were used (5453.91, 5552.90 and $7103.00 \mathrm{~m} / \mathrm{z}$ ). Furthermore, this second dendrogram was validated by PLS-DA and SVM algorithms. K-fold $(\mathrm{k}=10)$ was applied achieving $95.55 \%$ efficacy in both analyses.

In the single-step method, the peak matrix built with 5 biomarkers was used as an input for PLS-DA and SVM analysis in order to achieve the discrimination of the 3 Cryptococcus species simultaneously. PLS-DA analysis could not classify correctly the three varieties at the same time due to the low $\mathrm{k}$-fold $(\mathrm{k}=10)$ values obtained. However, PCA performance prior to SVM allowed $98.46 \%$ correct classification of the three Cryptococcus species (Figure 3). The efficacy of the method was tested by $k$-fold $(k=10)$ cross validation analysis was above $95.0 \%$. (Figure 3, Table S3) 
Table 2. List of the 10 representative mass peaks of Cryptococcus spp. Identified as potential biomarkers. These peaks were used for the construction of dendrograms and PLS-DA and SVM models. The 5 peaks marked with asterisks $\left(^{*}\right)$ were selected for the simplified models. CV=Coefficient of Variation.

\begin{tabular}{|c|c|c|c|c|c|c|c|c|c|c|}
\hline Mass $(\mathrm{m} / \mathrm{z})$ & $\begin{array}{l}Z \\
Z \\
\Xi \\
\\
0 \\
0 \\
0 \\
0 \\
\infty \\
0 \\
0 \\
D \\
D \\
D\end{array}$ & 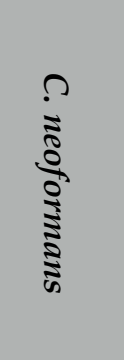 & 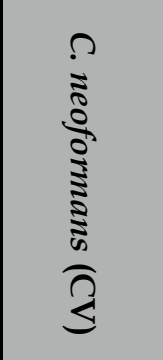 & 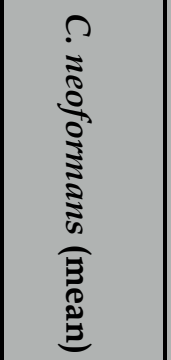 & 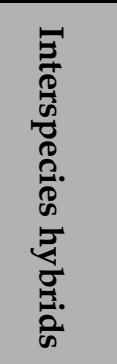 & 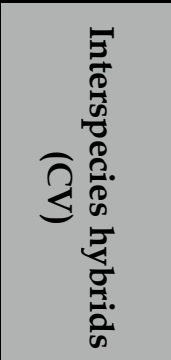 & 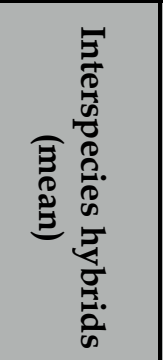 & 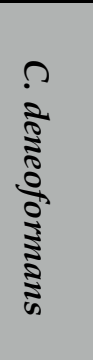 & 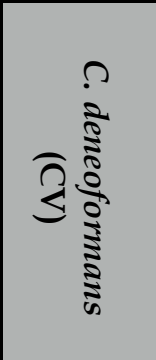 & 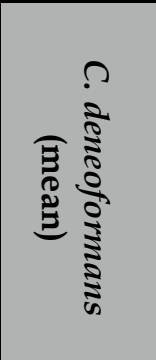 \\
\hline 2488.07 & 54 & $30 / 34$ & $88.749 \%$ & 4.401 .767 & $24 / 24$ & $69.617 \%$ & 2.811 .789 & $0 / 7$ & - & - \\
\hline 2842.14 & 53 & $29 / 34$ & $78.206 \%$ & 2.202 .128 & $24 / 24$ & $79.602 \%$ & 2.235 .196 & $0 / 7$ & - & - \\
\hline *3084.11 & 55 & $31 / 34$ & $98.458 \%$ & 7800.06 & $24 / 24$ & $89.283 \%$ & 5.969 .393 & $0 / 7$ & - & - \\
\hline *5453.91 & 27 & $1 / 34$ & $0.0 \%$ & 72.906 & $23 / 24$ & $65.081 \%$ & 731.902 & $3 / 7$ & $12.654 \%$ & 748.872 \\
\hline *5552.90 & 27 & $1 / 34$ & $0.0 \%$ & 558.307 & $23 / 24$ & $73.624 \%$ & 1.418 .905 & $3 / 7$ & $47.3 \%$ & 2.763 .278 \\
\hline 6576.08 & 23 & $0 / 34$ & - & - & $16 / 24$ & $63.172 \%$ & 457.978 & $7 / 7$ & $56.698 \%$ & 685.58 \\
\hline *6688.67 & 57 & $34 / 34$ & $95.69 \%$ & 4.420 .907 & $23 / 24$ & $88.388 \%$ & 3.556 .217 & $0 / 7$ & - & - \\
\hline *7103.01 & 31 & $1 / 34$ & $0.0 \%$ & 24.32 & $23 / 24$ & $122.759 \%$ & 1.484 .767 & $7 / 7$ & $52.14 \%$ & 4.155 .275 \\
\hline 7830.42 & 18 & $0 / 34$ & - & - & $11 / 24$ & $46.494 \%$ & 719.13 & $7 / 7$ & $39.831 \%$ & 449.704 \\
\hline 8636.24 & 43 & $19 / 34$ & $101.061 \%$ & 2.887 .856 & $24 / 24$ & $87.315 \%$ & 1.832 .722 & $0 / 7$ & - & - \\
\hline
\end{tabular}




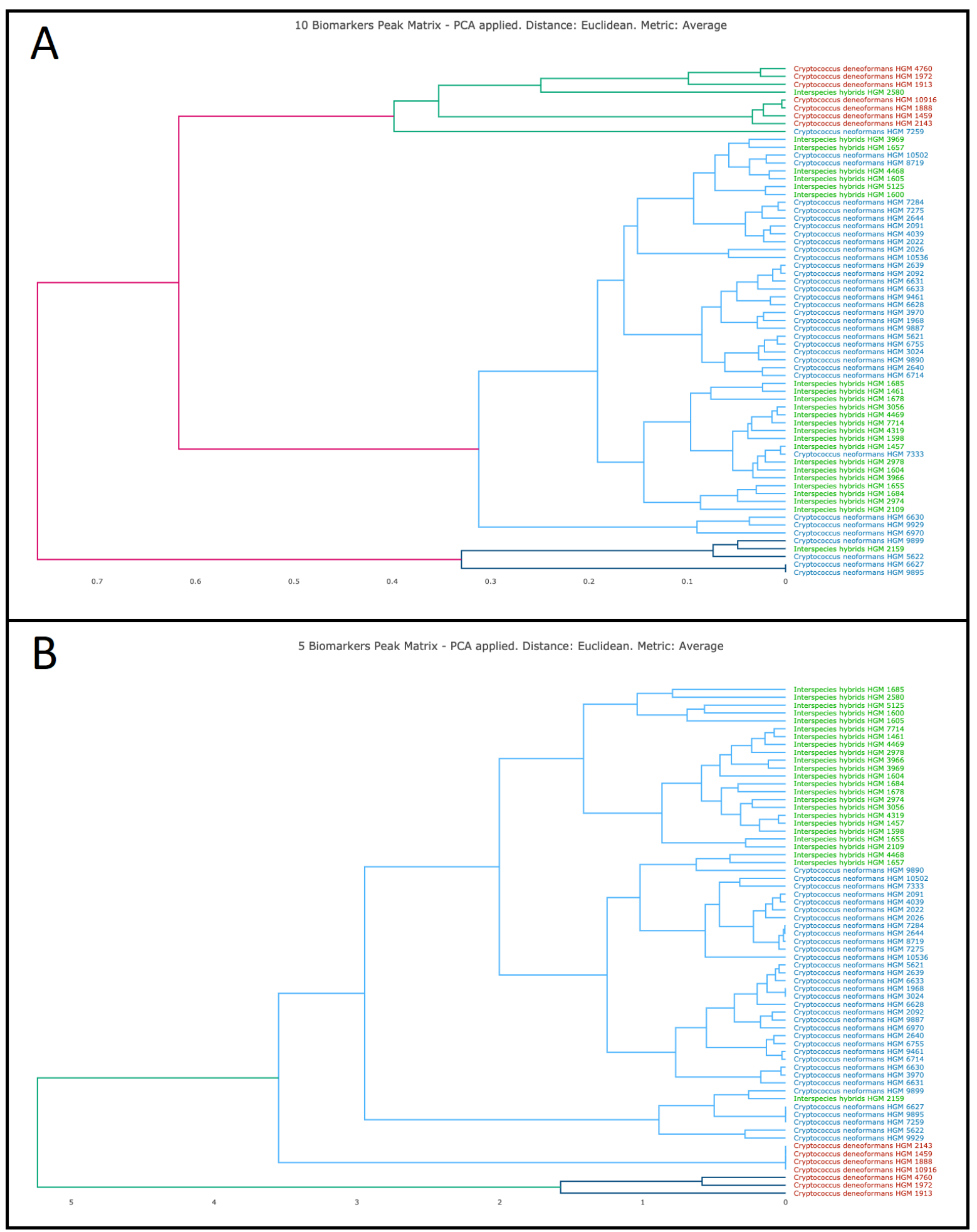

Figure 2. Clustering of 65 Cryptococcus isolates included in this study in a two-step approach. Five isolates could not be recovered from culture for further analysis. (a) Clustering using 10 biomarker peaks and PCA, b) Clustering using 5 biomarker peaks and PCA. 


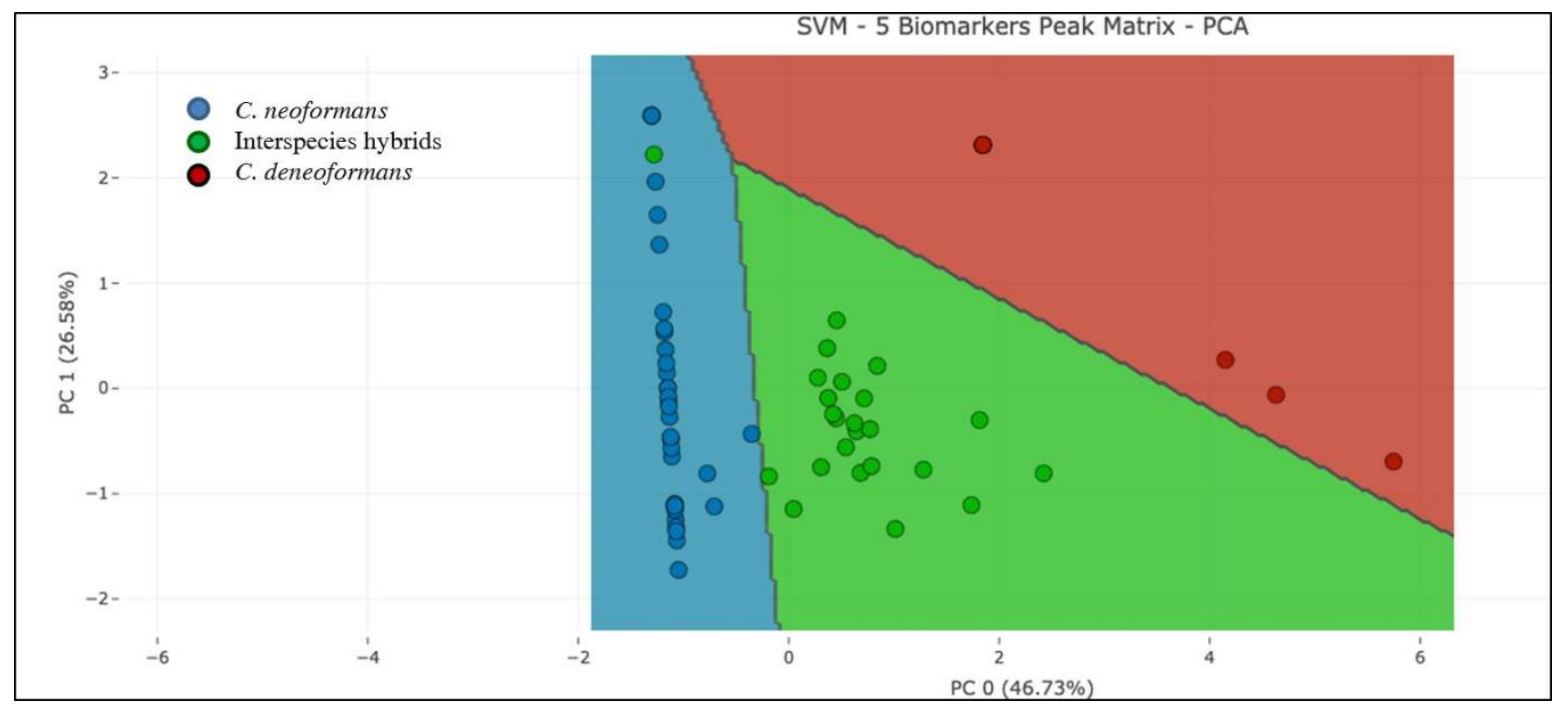

Figure 3. Classification of the three Cryptococcus species by SVM in the one-step approach, using 5 biomarker peaks.

Table 3. Differentiation of the analyzed Cryptococcus species based on the absence/presence of biomarker peaks. Figures indicate the percentage (\%) of isolates showing the indicated peak.

\begin{tabular}{|l|c|c|c|c|c|c|}
\hline \multicolumn{1}{|c|}{$\mathrm{m} / \mathrm{z}$} & $\mathbf{2 8 4 2 . 1 4}$ & $\mathbf{3 0 8 4 . 1 1}$ & $\mathbf{6 5 7 6 . 0 8}$ & $\mathbf{6 6 8 8 . 6 7}$ & $\mathbf{7 1 0 3 . 0 1}$ & $\mathbf{8 6 3 6 . 2 4}$ \\
\hline C. deneoformans & 0 & 0 & 100 & 0 & 100 & 0 \\
\hline C. neoformans & 85.3 & 91.2 & 0 & 100 & 0 & 55.9 \\
\hline Interspecies hybrids & 100 & 100 & 66.7 & 95.8 & 4.3 & 100 \\
\hline
\end{tabular}

As a result of this analysis, a visual method for the differentiation of the analyzed Cryptococcus species can be applied based on the presence of the $6688.67 \mathrm{~m} / \mathrm{z}$ peak in the C. neoformans isolates and their absence in C. deneoformans isolates, where the peaks $6576.08 \mathrm{~m} / \mathrm{z}$ and $7103.01 \mathrm{~m} / \mathrm{z}$ could be detected. On the other hand, both sets of peaks are present in the interspecies hybrids although some of them $(2842.14,3084.11$ and $8636.24 \mathrm{~m} / \mathrm{z})$ were detected in $100 \%$ of the spectra from this species (Table 3). The visual detection of these biomarker peaks could provide a rapid and accurate identification of the Cryptococcus species prior to a more in-depth peak analysis using ad-hoc software.

\section{Discussion}

Accurate identification of Cryptococcus species within the C. neoformans complex provides valuable information about their epidemiology, sensitivity to commonly used antifungal drugs or virulence. Our results show that discrimination among the three Cryptococcus species analyzed-C. deneoformans, C. neoformans and interspecies hybrids- can be performed successfully using MALDI-TOF MS and peak analysis.

The implementation of the in-house database built in our laboratory allowed $100 \%$ correct species-level identification of the $25 \mathrm{C}$. deneoformans and C. neoformans isolates used to challenge it. Apart from the reliable identification of the analyzed Cryptococcus species, the in-house library also provided high confidence identifications in $63.6 \%$ of the cases. Furthermore, these results showed consistency along the 10 top identifications provided by the mass spectrometry instrument, even for 
the hybrids. This fact is of great importance in the routine of the microbiology laboratory in order to transfer reliable information to the clinicians.

The results obtained are in agreement with those obtained by other authors [21-23]. However, the in-house library did not provide enough discrimination between the above-mentioned species and the interspecies hybrids. This goal was only fulfilled completely when peak analysis was performed and the three Cryptococcus species analyzed in this study distinctively clustered together. Other authors have provided species-level discrimination in $98.1-100 \%$ of the cases [21, 23, 27]. Although some of these studies were performed on higher number of isolates, our results also reflect the improvements made on the commercial database during the last years.

The available commercial database has demonstrated to provide high species-level resolution for $C$. deneoformans and C. neoformans $-76.0 \%$ - although score values $<1.7$ were obtained in $21.0 \%$ of the cases and species-level identification was not provided for 2 C. deneoformans isolates. These data supported the need of building expanded databases. However, even improvements in the reference databases proved not to be enough to differentiate the interspecies hybrids. This may be due to the algorithms used by the mass spectrometry instrument for species assignment and to the fact that the hybrids show peaks present of both parental species. Therefore, peak analysis using ad-hoc software was performed. A list of 10 biomarker peaks was achieved as the input for species classification. The implementation of PLS-DA analysis in a two-step approach allowed the discrimination of $C$. deneoformans isolates in the first place and, subsequently, the correct classification of C. neoformans isolates and the interspecies hybrids in $96.92 \%$ of the cases. Furthermore, the accuracy of this method increased when the number of biomarker peaks used was reduced to the five most discriminative ones $(98.46 \%)$.

In order to simply the analysis, a one-step method was applied in order to classify the three species simultaneously. In this case, PLS-DA provided correct classification in less than $75.0 \%$ of the cases but the application of SVM after PCA analysis allowed $96.92 \%$ correct discrimination of the analyzed isolates. This analysis provided a set of species-specific peaks for the Cryptococcus species within the $C$. neoformans complex that may be detected by visual inspection, representing a rapid and inexpensive approach for their discrimination.

\section{Conclusions}

Our results demonstrate the usefulness of MALDI-TOF MS and peak analysis when applied in the microbiology laboratory for rapid and reliable identification of non-Candida yeasts. Although the updated commercial library provided correct species-level identification for a high number of $C$. deneoformans and C. neoformans isolates, the identification of these species was missing or not reliable in $20.5 \% 18.2 \%$ of the cases, respectively. Moreover, the detection of the interspecies hybrids is not possible with the Biotyper database. However, the expanded in-house library allowed correct species-level identification for all $C$. deneoformans and $C$. neoformans, either by conventional identification with MALDI-TOF MS or by peak analysis. The interspecies hybrids required hierarchical clustering for their correct identification since their close relatedness with the other species made it difficult for MALDI-TOF to differentiate them from the other two species in a routine manner. This approach and the detection of species-specific peaks are recommended for the reliable discrimination of the three analyzed species.

\section{Supplementary Materials:}

Table S1. Pre-treatment of raw data spectra for the biomarkers search.

Table S2. Parameters applied for the construction of the peak matrix.

Table S3. Score and validation of (A) the SVM analysis applied to the two-step model with 5 biomarkers and (B) PLS-DA applied to the one-step model with 5 biomarkers. $K=10$

Figure S1. ROC Curve of MS peaks of Cryptococcus spp. according to the criteria for biomarkers search.

Figure S2. Discrimination between C. neoformans and the interspecies hybrids using the two-step classification method. 
Author Contributions: Margarita E. Zvezdanova: Methodology (lead); formal analysis (lead); writing - original draft (supporting) - review and editing (equal). Manuel J. Arroyo: Software (lead); writing - review and editing (equal). Gema Méndez: Software (equal); review and editing (equal). Jesús Guinea: Conceptualization (supporting); formal analysis (supporting); writing - review and editing (equal). Luis Mancera: Software (lead); writing - review and editing (equal). Patricia Muñoz: Writing - review and editing (equal). Belén Rodríguez-Sánchez: Conceptualization (lead); formal analysis (lead); writing - review and editing (lead). Pilar Escribano: Conceptualization (lead); formal analysis (lead); writing - review and editing (lead).

Funding: This study has been supported by the Miguel Servet Program (ISCIII-MICINN CP14/00220) and by the projects PI16/01012 (PE) and PI18/00997 (BRS) from the Health Research Fund (FIS) of the Carlos III Health Institute (ISCIII), Madrid, Spain, partially financed by the by the European Regional Development Fund (FEDER) 'A way of making Europe.' BRS (CPII19/00002), PE (CPI15/00115) and JG (CPII15/00006) are recipients of a Miguel Servet contract supported by the FIS program. The funders had no role in the study design, data collection and analysis, decision to publish, or preparation of the manuscript.

Acknowledgments: The authors want to thank Álvaro Gómez-González for his assistance with Bruker Daltonics databases.

Conflicts of Interest: The authors declare no conflict of interest.

\section{References}

1. Kwon-Chung KJ, Fraser JA, Doering TL, Wang Z, Janbon G, Idnurm A. et al. Cryptococcus neoformans and Cryptococcus gattii, the etiologic agents of cryptococcosis. Cold Spring Harb Perspect Med. 2014;4:a019760.

2. D'Souza CA, Kronstad JW, Taylor G, Warren R, Yuen M, Hu G. et al. Genome variation in Cryptococcus gattii, an emerging pathogen of immunocompetent hosts. mBio 2011;2:e00342-10.

3. Trilles L, Meyer W, Wanke B, Guarro J, Lazéra M. Correlation of antifungal susceptibility and molecular type within the Cryptococcus neoformans/C. gattii species complex. Med Mycol. 2012;50:328-32.

4. Chong HS, Dagg R, Malik R, Chen S, Carter D. In vitro susceptibility of the yeast pathogen Cryptococcus to fluconazole and other azoles varies with molecular genotype. J Clin Microbiol. 2010;48:4115-20.

5. Kwon-Chung KJ, Bennett JE. Epidemiologic differences between the two varieties of Cryptococcus neoformans. Am J Epidemiol. 1984;120:123-30.

6. Iqbal N, DeBess EE, Wohrle R, Sun B, Nett RJ, Ahlquist AM, et al. Correlation of genotype and in vitro susceptibilities of Cryptococcus gattii strains from the Pacific Northwest of the United States. J. Clin. Microbiol. 2010;48:539-544.

7. Perfect JR, Bicanic T. Cryptococcosis diagnosis and treatment: What do we know now. Fungal Genet Biol. 2015;78:49-54.

8. Feng X, Yao Z, Ren D, Liao W. Simultaneous identification of molecular and mating types within the Cryptococcus species complex by PCR-RFLP analysis. J Med Microbiol. 2008;57:1481-90.

9. Pakshir K, Fakhim H, Vaezi A, Meis JF, Mahmoodi M, Zomorodian K. et al. Molecular epidemiology of environmental Cryptococcus species isolates based on amplified fragment length polymorphism. J Mycol Med. 2018;28:599-605.

10. Hanafy A, Kaocharoen S, Jover-Botella A, Katsu M, Iida S, Kogure T, Gonoi T, Mikami Y, Meyer W. Multilocus microsatellite typing for Cryptococcus neoformans var. grubii. Med Mycol. 2008;46:685-96.

11. Meyer W, Aanensen DM, Boekhout T, Cogliati M, Diaz MR, Esposto MC, et al. Consensus multi-locus sequence typing scheme for Cryptococcus neoformans and Cryptococcus gattii. Med Mycol. 2009;47:561-70.

12. Franzot SP, Salkin IF, Casadevall A. Cryptococcus neoformans var. grubii: separate varietal status for Cryptococcus neoformans serotype A isolates. J. Clin. Microbiol. 1999;37:838-840.

13. Meyer W, Castañeda A, Jackson S, Huynh M, Castañeda E. Molecular typing of IberoAmerican Cryptococcus neoformans isolates. Emerg Infect Dis. 2003;9:189-95.

14. Hagen F, Hare Jensen R, Meis JF, Arendrup MC. Molecular epidemiology and in vitro antifungal susceptibility testing of 108 clinical Cryptococcus neoformans sensu lato and Cryptococcus gattii sensu lato isolates from Denmark. Mycoses. 2016;59:576-84 
15. Hagen F, Khayhan K, Theelen B, Kolecka A, Polacheck I, Sionov E, et al. Recognition of seven species in the Cryptococcus gattii/Cryptococcus neoformans species complex. Fungal Genet Biol. 2015;78:16-48.

16. De Carolis E, Vella A, Vaccaro L, Torelli R, Posteraro P, Ricciardi W, et al. Development and validation of an in-house database for matrix-assisted laser desorption ionization-time of flight mass spectrometry-based yeast identification using a fast protein extraction procedure. J Clin Microbiol. 2014;52:1453-8.

17. Jamal WY, Ahmad S, Khan ZU, Rotimi VO. Comparative evaluation of two matrix-assisted laser desorption/ionization time-of-flight mass spectrometry (MALDI-TOF MS) systems for the identification of clinically significant yeasts. Int J Infect Dis. 2014;26:167-70

18. Fraser M, Brown Z, Houldsworth M, Borman AM, Johnson EM. Rapid identification of 6328 isolates of pathogenic yeasts using MALDI-ToF MS and a simplified, rapid extraction procedure that is compatible with the Bruker Biotyper platform and database. Med Mycol. 2016;54:80-8.

19. Spanu T, Posteraro B, Fiori B, D'Inzeo T, Campoli S, Ruggeri A, et al. Direct maldi-tof mass spectrometry assay of blood culture broths for rapid identification of Candida species causing bloodstream infections: an observational study in two large microbiology laboratories. J Clin Microbiol. 2012;50:176-9.

20. Bader O, Weig M, Taverne-Ghadwal L, Lugert R, Gross U, Kuhns M. Improved clinical laboratory identification of human pathogenic yeasts by matrix assisted laser desorption ionization time-of-flight mass spectrometry. Clin Microbiol Infect 2011;17:1359-1365.

21. McTaggart LR, Richardson SE, Seah C, Hoang L, Fothergill A, Zhang SX. Rapid identification of Cryptococcus neoformans and Cryptococcus gattii by matrix-assisted laser desorption ionization- time of flight mass spectrometry. J. Clin. Microbiol. 2011;49:3050 -3053.

22. Firacative C, Trilles L, Meyer W. MALDI-TOF MS Enables the Rapid Identification of the Major Molecular Types within the Cryptococcus neoformans/C. gattii Species Complex. PLoS One. 2012;7:e37566.

23. Posteraro B, Vella A, Cogliati M, De Carolis E, Florio AR, Posteraro P, Sanguinetti M, Tortorano AM. Matrix-assisted laser desorption ionization-time of flight mass spectrometry-based method for discrimination between molecular types of Cryptococcus neoformans and Cryptococcus gattii. J Clin Microbiol. 2012;50:2472-6.

24. Katsu M, Kidd S, Ando A, Moretti-Branchini ML, Mikami Y, Nishimura K. et al. The internal transcribed spacers and 5.8S rRNA gene show extensive diversity among isolates of the Cryptococcus neoformans species complex. FEMS Yeast Res. 2004;4:377-88.

25. Guinea J, Hagen F, Peláez T, Boekhout $T$, Tahoune H, Torres-Narbona M. et al. Antifungal susceptibility, serotyping, and genotyping of clinical Cryptococcus neoformans isolates collected during 18 years in a single institution in Madrid, Spain. Med Mycol. 2010;48:942-8.

26. Zvezdanova ME, Escribano $P$, Ruiz A, Martínez-Jiménez MC, Peláez T, Collazos A, et al. Increased species-assignment of filamentous fungi using MALDI-TOF MS coupled with a simplified sample processing and an in-house library. Med Mycol. 2018. doi: 10.1093/mmy/myx154.

27. Siqueira LPM, Gimenes VMF, de Freitas RS, Melhem MSC, Bonfietti LX, da Silva AR Jr, et al. Evaluation of Vitek MS for Differentiation of Cryptococcus neoformans and Cryptococcus gattii Genotypes. J Clin Microbiol. 2019;57(1). pii: e01282-18. 